\title{
The psychophysics of remembered duration
}

\author{
J. GREGOR FETTERMAN \\ Indiana University-Purdue University, Indianapolis, Indiana
}

\begin{abstract}
Pigeons were trained on a psychophysical choice task to make one response after a 2-sec signal and a different response after a 10-sec signal. Delayed dimensional control was assessed by presenting durations intermediate to the short and long signals and by introducing delays between the signals and choice opportunities. In Experiment 1, choices after intermediate durations were not reinforced; in Experiment 2, one choice was reinforced after the three shortest durations and another was reinforced after the three longest durations. In Experiment 1, the slopes of the psychophysical functions decreased with increases in delays, but the decrease in stimulus control was not unbiased; choice probabilities decreased for longer durations, but did not increase for shorter durations. Experiment 2 revealed the same generalized loss of stimulus control on the temporal dimension, but not the same pattern of bias; temporal control was relinquished equally for shorter and longer durations. These results are evaluated in the context of the subjective shortening model of remembered duration (Spetch \& Wilkie, 1983) and Staddon's theory of timing and remembering (Staddon, 1984).
\end{abstract}

Spetch and Wilkie $(1982,1983)$ first reported on a phenomenon that has since received a good deal of empirical and theoretical attention. In their experiments, pigeons were trained on a task under which one choice was reinforced after a short-duration signal (e.g., $2 \mathrm{sec}$ ) and a different choice was reinforced after a long-duration signal (e.g., $10 \mathrm{sec}$ ). The data of interest were obtained from a test of working memory for stimulus duration in which delays were interposed between the offset of the signal and presentation of the choice alternatives. Overall accuracy declined with increasing delay, as expected. More importantly, changes in stimulus control after the short and long signals were strikingly different. There was very little change in accuracy across delays on shortsignal trials, whereas accuracy on long-signal trials decreased dramatically, sometimes falling well below chance performance. This phenomenon, the choose-short effect, has proven to be remarkably robust, having been observed in numerous experiments and in different laboratories (e.g., see a review by Spetch \& Rusak, 1992). Related effects have been observed with nontemporal stimuli, including response number (Fetterman \& MacEwen, 1989) and rate of alternation (Honig \& Spetch, 1988).

Following the original reports by Spetch and Wilkie, much of the relevant research has centered on researchers' attempts to identify the processes that under-

This research was supported by grants from NSF (BNS 9021562) and NIMH (RO1 MH48253). Some of the data were reported at the 1993 meeting of the Midwestern Psychological Association, Chicago. Alan Stubbs provided many helpful comments on a draft of the manuscript. Correspondence and requests for reprints should be addressed to J. G. Fetterman, Department of Psychology, LD 3124, 402 N. Blackford St., Indianapolis, IN 46202-3275 (e-mail: itnk100@indyvax. iupui.edu). lie temporal working memory and to delimit the conditions under which the biased forgetting effect does and does not occur. For instance, Spetch and Wilkie (1983) hypothesized that the asymmetries of forgetting along the temporal dimension resulted from a process termed subjective shortening. According to this account, remembered duration grows shorter and shorter throughout the delay interval so that, at the time of the test, the subjective duration of a signal appears much shorter than its nominal value. This account implies that animals retain an analogical code for duration and evaluate the duration of the prior signal retrospectively (Spetch \& Rusak, 1992); there is indeed compelling evidence for this view of delayed temporal control (e.g., Wilkie \& Willson, 1990).

Alternatively, others (e.g., Kraemer, 1991; Kraemer, Mazmanian, \& Roberts, 1985) have argued that the choose-short bias reflects the probabilistic forgetting of a categorical memory code ("short" versus "long") coupled with a tendency to default to the short choice once the code has been completely lost from working memory. This latter assumption is consistent with a no-sample bias observed by Spetch and Wilkie (1983) and others (e.g., Church, 1980; Fetterman \& MacEwen, 1989); when probe trials are given in which the choices are offered without a prior sample, subjects tend to respond to the short choice alternative.

Discussions concerning the characteristics of temporal memory appear to center on the analogical/categorical distinction and on whether subjects rely on prospective or retrospective cues. The prospective/retrospective coding dichotomy has received considerable attention in the working-memory literature (Honig \& Thompson, 1982). Subjects may maintain a memory code based on the prior sample (retrospective code), or they might adopt a "forward-looking" strategy by maintaining a code that is representative of the correct response alternative (prospective code). The analogical/categorical 
and prospective/retrospective distinctions are not completely orthogonal, and both have been addressed in recent experiments on temporal working memory. For instance, Spetch and Sinha (1989) trained pigeons on a delayed symbolic-matching-to-sample (DSMTS) task with duration samples and presented occasional trials in which the target duration was preceded by a "presample" duration. The pigeons' choices reflected a tendency to sum the durations of presample and target stimuli, consistent with predictions of an analogical/retrospective coding model and incompatible with a categorical/ prospective model of temporal coding. Wilkie and Willson (1990) reported similar results.

Some procedures, however, appear to favor a different coding strategy. Grant and Spetch (1991) trained pigeons on a successive matching-to-sample task with duration as the discriminative cue. With the successive task, a single test stimulus is presented after the sample; one test stimulus is positive after the short sample, and a different test stimulus is positive after the long sample. Discrimination is indexed by the difference in response rates to the test stimulus on positive and negative trials. Grant and Spetch measured discrimination separately for short- and long-sample trials and observed a decline in accuracy with increases in delay that was similar for both sample types; that is, the animals did not tend to "respond short." Grant and Spetch argued that this result implies a difference in coding strategy between choice and successive matching procedures, and they concluded that pigeons use a prospective code in the latter context versus a retrospective code in the former.

Other modifications of the DSMTS procedure also affect the character of the inferred memory code. For instance, a pigeon might be trained to peck a horizontal comparison after 2-sec houselight and red keylight samples, and to peck a vertical comparison after a 10 -sec houselight and a green keylight. Under this many-to-one (MTO) mapping procedure, the forgetting functions after short and long samples are nearly identical (Grant \& Spetch, 1993; Santi, Bridson, \& Ducharme, 1993). One explanation of this finding presumes that the MTO mapping induces prospective coding strategies and that such strategies are incompatible with the subjective shortening process. Seemingly minor modifications of the basic task may likewise eliminate the disparity in retention on short- and long-sample trials. Spetch and Rusak (1992; see also Fetterman \& MacEwen, 1989) found that the choose-short effect could be eliminated by clearly demarcating intertrial interval (ITI) and delay periods. For example, when the houselight was off during ITIs and delays, the pigeons showed the typical choose-short bias, but the bias was eliminated when the houselight was on during one period and off during the other. It is not clear how this subtle procedural change might alter the pigeon's working-memory strategy.

Although a variety of clever variations on the basic DSMTS task have been used to investigate temporal working memory, very little effort has been devoted to addressing questions related to the psychophysics of de- layed temporal control, and such questions seem fundamental to the issues at hand. Do changes along the temporal continuum produce corresponding changes in pigeons' choice responses? Further, if delayed choices are related to sample duration, is that relation consistent with the subjective shortening process? These questions seem basic to an understanding of the qualitative and quantitative features of remembered duration.

The subjective shortening model holds that the remembered value of the signal corresponds to the nominal sample duration discounted by some (unspecified) amount, with the magnitude of the discounting over time based on the rate of the subjective shortening process. Note that although nominal and remembered durations are not held to be in strict accord, the hypothesized memory code bears an analogical relation to the sample, and choices should be sensitive to changes in sample duration, but in a biased way. Other accounts (e.g., Kraemer et al., 1985) presume that the temporal characteristics of the signal are lost once the sample has been "recoded" onto some other dimension. For instance, a subject might label the signal duration "short" or "long" (a categorical code), and such a code would contain no information specific to the value of the sample, just as describing a person as short or tall provides very little information about the true height of the individual in question. Similarly, a subject that forms a prospective code (e.g. "peck the red comparison") has no direct access to the duration of the prior signal.

The experiments in this article were conducted to provide more extensive information about the psychophysics of temporal memory than that provided in an early report by Spetch and Wilkie (1983). The method of constant stimuli was used to assess control along the temporal dimension across variable delays between the temporal sample and choice. Standard analyses were employed to assess changes in the psychophysical functions relating choices to signal duration, and these changes were compared with different accounts of temporal memory.

\section{EXPERIMENT 1}

In the first experiment, pigeons were trained to make one response after a short signal duration and a different response after a long signal duration. All the birds then received a series of retention tests in which the onset of the choice key stimuli was delayed from the end of the signal. In the first test, two signal values $(2$ and $10 \mathrm{sec}$ ) were used; in the remaining tests, durations intermediate to the shortest and longest values were included. The purpose was to assess whether the delayed choices were related to changes in signal duration and to determine whether changes in performance along the temporal continuum were consistent with the subjective shortening account.

\section{Method}

Subjects. The subjects were 4 adult male Silver King pigeons (Columba livia), maintained at $85 \%$ of their free-feeding weights. 
The birds were maintained on a 12:12-h light:dark cycle with light onset at 7:00 a.m. The pigeons received unlimited access to water and grit in their home cages. The subjects were experimentally naive at the beginning of the experiment.

Apparatus. The experimental enclosure was a standard BRSLVE three-key pigeon chamber; the work space measured $32 \mathrm{~cm}$ high $\times 34 \mathrm{~cm}$ wide $\times 34 \mathrm{~cm}$ deep. The keys were accessible through 2-cm circular openings in the work panel on the front wall, with the center of the openings spaced $6.3 \mathrm{~cm}$ apart, $24 \mathrm{~cm}$ above the chamber floor. A force of approximately $0.15 \mathrm{~N}$ was required to operate each of the keys. The feeder opening was located directly below the center response key and measured $5 \mathrm{~cm}$ on all dimensions; the bottom of the feeder opening was $10 \mathrm{~cm}$ above the chamber floor. White noise served to mask extraneous sounds; additional masking and ventilation were provided by an exhaust fan attached to the chamber wall. Experimental events were scheduled and recorded by an IBM PC and interface located in an adjacent room.

Procedure. After magazine training, the pigeons were exposed to an autoshaping procedure (Brown \& Jenkins, 1968) under which they received 90 trials per session for five sessions. A trial commenced with the onset of an amber light behind one of the pecking keys; if no peck occurred within $5 \mathrm{sec}$ of the onset of the signal, the light was turned off and the food hopper was raised for $3 \mathrm{sec}$; a peck to the lit key produced food immediately. Food presentations were followed by an ITI that lasted for $30 \mathrm{sec}$. The different keylights were intermixed in a quasi-random order across trials, and each key was lit 30 times in each session. This procedure produced reliable pecking on all keys by all birds within four sessions.

Once keypecking was established, all the birds were placed on a symbolic-matching-to-sample procedure (SMTS), under which the duration of the center keylight served as the discriminative stimulus. Trials began with the illumination of the center key by amber light. The light remained on for $2 \mathrm{sec}$ (short) or $10 \mathrm{sec}$ (long). The durations were presented in a quasi-random order, each with a probability of .50 . Following either duration, the center keylight was turned off and the side keys were illuminated, one by a red light and the other by a green light. The position of the red and green lights alternated randomly across trials, subject to the restriction that each color appear equally often on the left and right keys. For 2 birds (P-39 and P-02), a peck on the red key was correct after the short signal and a peck on the green key was correct after the long signal. This arrangement was reversed for the other 2 pigeons ( $\mathrm{P}-53$ and $\mathrm{P}-04$ ). Correct responses produced $3-\mathrm{sec}$ access to mixed grain followed by a $30-\mathrm{sec}$ ITI during which all lights were off. Incorrect responses produced the ITI directly. A correction procedure, whereby sample durations were repeated across trials until a subject responded to the correct choice alternative, was in effect during the first 10 sessions of training; a noncorrection procedure was used thereafter. Sessions ended after 60 trials.

Once the discrimination was acquired to a criterion of $80 \%$ correct or better on 5 consecutive sessions (this took between 15 and 26 sessions for different pigeons), the probability of reinforcement for correct choices was reduced from 1.0 to .50 . Reinforcement was arranged equally for correct choices of the short and long alternatives, according to the method devised by Stubbs (1976). Reinforcers were randomly assigned for correct red-key or green-key responses with a probability of .50 for either key, and a reinforcer had to be collected before another could be assigned. Reinforcement was provided when the pigeon correctly responded on the designated key. Correct responses on the other key simply produced the ITI, as did all incorrect responses. The pigeons received 20 additional sessions of training with partial reinforcement of choices before delay testing was begun.

The first retention test was conducted with the short-and longsignal values only. As before, the trials began with the onset of the center keylight signal, which lasted for 2 or $10 \mathrm{sec}$. On $40 \%$ of the trials, the choice keylights were turned on immediately after the center keylight was turned off ( 0 -sec delay). On the remaining trials, a delay of 2,5 , or $15 \mathrm{sec}$ was interposed between the offset of the center keylight and the onset of the choice keylights; each delay was presented on $20 \%$ of the trials. The sequencing of delays and signal durations was arranged in a quasi-random order, and each delay occurred equally after the short and long signals. All lights were off during the delay intervals and ITIs. Correct responses were reinforced with a probability of .50 , as noted above, and ITIs lasted $30 \mathrm{sec}$. Sessions ended after 80 trials, and delay testing lasted for 10 sessions.

The birds were then returned to their original discriminations, without delays, for 10 sessions and were then tested with probe durations intermediate to the values of the short and long durations. Four probe durations, spaced $0.14 \mathrm{log}$ units apart, were used; the probe set included durations of $2.75,3.8,5.25$, and $7.24 \mathrm{sec}$. Probe durations were presented on one half of the trials, with trials equally distributed among the four values. Choices on probe trials were not reinforced and simply produced the ITI. The short $(2 \mathrm{sec})$ and long $(10 \mathrm{sec})$ signals were presented on the remaining trials, and every correct response was reinforced. Thus, as in earlier conditions, reinforcement was received on approximately $50 \%$ of the trials. Sessions ended after 90 trials and the initial phase of psychophysical testing lasted five sessions.

All the pigeons were then given two delay tests that included both training and probe durations. In the first test, which began immediately after the first phase of psychophysical testing, delays of $0,2,5$, and $15 \mathrm{sec}$ were intermixed within sessions. The 0 -sec delay occurred on $40 \%$ of the trials, and the remaining trials were equally divided among the three nonzero delays. The second delay test was conducted after data reported as Experiment 2 were collected. All the birds were returned to the original discrimination task ( 2 vs. $10 \mathrm{sec}$ ) without delays for 30 sessions before the second test series was conducted. In the second test, delays of $0,3,6,12$, and $18 \mathrm{sec}$ were intermixed within each session. The 0 -sec delay occurred on $40 \%$ of the trials, and the remaining trials were equally divided among the four nonzero delays. Each delay test lasted for 30 sessions, and the sessions ended after 120 trials. Training and probe durations were presented in the manner described previously; responses on probe duration trials were not reinforced, and all correct responses on training duration trials were reinforced. Durations and delays were presented in a quasi-random order, subject to the restriction that each duration and delay combination occurred at least once within each block of 40 trials.

\section{Results and Discussion}

Figure 1 shows the outcome of the two stimulus delay tests; the data are averaged over the 4 pigeons, but are highly representative of all birds. The top panel depicts the choose-short effect in the usual manner by showing the probability of responding correctly after the short signal (filled circles) and after the long signal (unfilled circles) as a function of test delay. The asymmetrical changes in accuracy after short and long signals are readily apparent. The bottom panel shows the changes in performance in a psychophysical format by presenting the probability of calling the signal "long," with signal duration on the abscissa and delay as the parameter. Here, biased forgetting is indicated by the asymmetrical changes in the delay functions, evident at the 15-sec test delay; probability values for the longer signal decrease toward .50 , but do not show a corresponding increase after the shorter signal. 

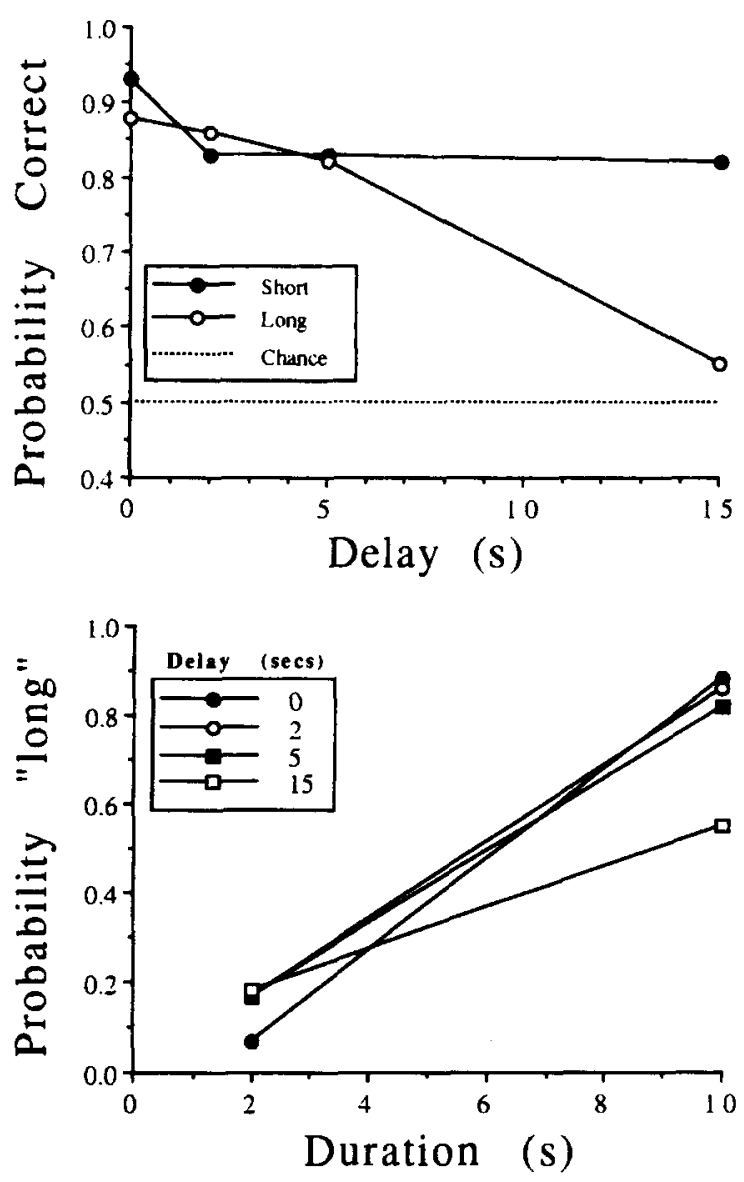

Figure 1. The top panel shows the probability of responding correctly as a function of delay after the short signal (filled circles) and after the long signal (unfilled circles). The bottom panel shows the probability of selecting the long choice alternative as a function of signal duration with delay as the parameter. (The data represent performance averaged over 4 pigeons.)

Figures 2 and 3 show psychophysical functions across delays for all the birds for the first (Figure 2) and second (Figure 3) multiple-sample delay tests; the functions are based on all 30 sessions of delay testing for each test series. As is shown in the bottom panel of Figure 1, the dependent measure is the probability of responding that a signal was the long signal; signal duration is represented on the abscissa and delay is the parameter. The changes in the psychophysical functions across increasing delays are not symmetrical, as would be expected if stimulus control decreased in an unbiased way. Instead, the functions change in a manner consistent with the subjective shortening account. Slopes decreased with increasing delay, but the changes were not symmetrical about .50; probabilities decreased for longer durations, but did not show a corresponding increase for shorter durations. This asymmetry is evident for all the birds, but is clearer for some than for others. For instance, P-04 displayed a very strong asymmetry in both delay tests, as did P-02.

The asymmetrical changes in the ogival functions are the psychophysical signature of the choose-short effect, whereas the decrease in the slopes of the curves reflects a general loss of stimulus control along the temporal dimension. These generalized changes in stimulus control were assessed through a regression analysis. The independent and dependent measures were transformed to $z$ scores, and linear regressions were fitted to the data of each bird to assess the extent of the changes in temporal control and to establish whether choices were sensitive to changes along the temporal dimension at long test delays. Slopes decreased significantly across delays for the first $[F(3,9)=75.76, p<.05]$ and second $[F(4,12)=$ $40.67, p<.05]$ test series. However, slopes were significantly greater than zero $(p<.05)$ for all the pigeons at the longest delay for each series, indicating that performance was sensitive to changes in signal duration at the same time that choices were clearly biased toward the short response alternative.

Figure 4 summarizes the results shown in Figures 2 and 3 by providing measures of sensitivity $\left(A^{\prime}\right)$ and bias $\left(B^{\prime \prime}\right)$. These measures, described by Grier (1971) and Fetterman, Dreyfus, and Stubbs (1993), provide independent assessments of changes in sensitivity to the temporal dimension $\left(A^{\prime}\right)$ and of the tendency to respond to one choice more than the other $\left(B^{\prime \prime}\right)$. The $A^{\prime}$ statistic is highly correlated with overall accuracy and has a typical range between .50 (chance) and 1.0 (perfect discrimination). The $B^{\prime \prime}$ measure can vary between -1.0 and 1.0 , with a value of 0 indicating no bias. For the calculations reported here, negative values of $B^{\prime \prime}$ indicate a bias toward the short alternative, and positive values reflect a bias toward the long alternative. Because correct responses were defined in terms of the shortest $(2 \mathrm{sec})$ and longest $(10 \mathrm{sec})$ signal durations only, the computations do not include unreinforced probe trials involving intermediate signal durations.

The top panel of Figure 4 shows changes in the sensitivity measure $\left(A^{\prime}\right)$ across delays for the first (unfilled circles) and second (filled circles) test series. The data are means of the 4 pigeons. Sensitivity decreased significantly with increases in delay for the first $[F(3,9)=$ $10.53, p<.05]$ and second $[F(4,12)=10.14, p<.05]$ test series. The bottom panel shows the changes in the bias measure, $B^{\prime \prime}$. Bias scores are not shown for the 0 -sec delay because this measure is extremely variable when accuracy is high; small changes in performance produce wide fluctuations in the bias measure. Because so few errors were made at the 0 -sec delay, performance was, practically speaking, unbiased (see Fetterman \& MacEwen, 1989, for further details on this point). The changes in bias were consistent with the subjective shortening effect. Values of $B^{\prime \prime}$ decreased significantly with increasing delay for the first $[F(2,6)=5.61, p<.05]$ and second $[F(3,9)=3.92, p<.05]$ test series.

Figure 5 shows the points of subjective equality (PSEs) across delays for each pigeon for the first (top panel) and second (bottom panel) test series. The PSEs were estimated from the regression equations fitted to the psychophysical functions in Figures 2 and 3 . The PSE, or bisection point, can be interpreted as the psy- 

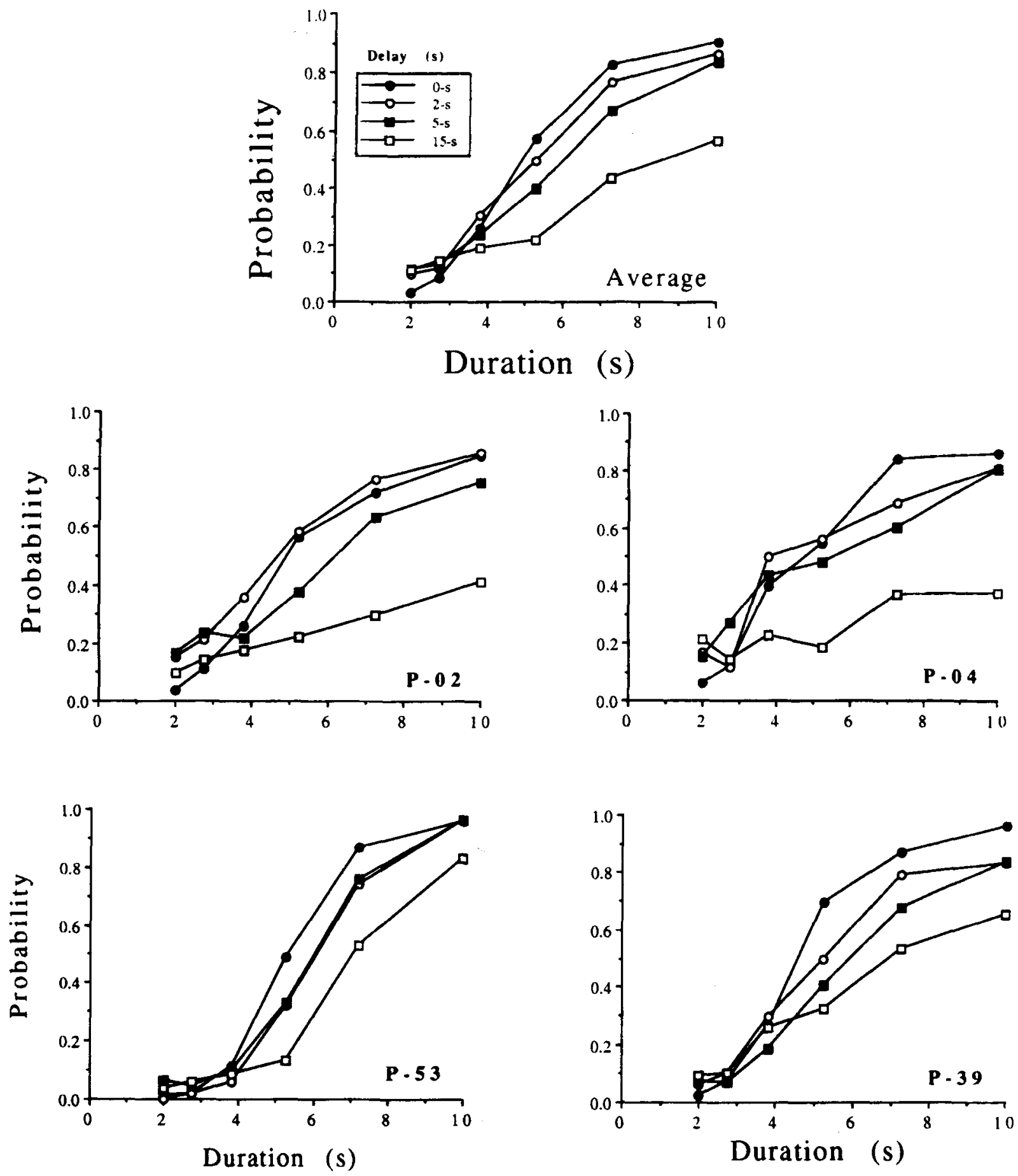

Figure 2. Psychometric functions relating the probability of responding to the long alternative to signal duration, with delay between signal offset and choice as the parameter. (The data were obtained from the first multiple-stimulus delay test.)

chological middle between two anchor stimuli (Gibbon, 1991); pigeons, rats, and humans are typically found to bisect at the geometric mean of two reinforced endpoints (e.g., Allan \& Gibbon, 1991; Church \& Deluty, 1977; Fetterman \& Killeen, 1992; Stubbs, 1968), indicated by the horizontal dashed lines in the figure. If remembered time undergoes changes like those suggested by the subjective shortening account, then PSEs should increase with increases in the test delay, precisely as shown in Figure 5.

The method of constant stimuli was used to generate psychophysical functions for duration; delays interposed 

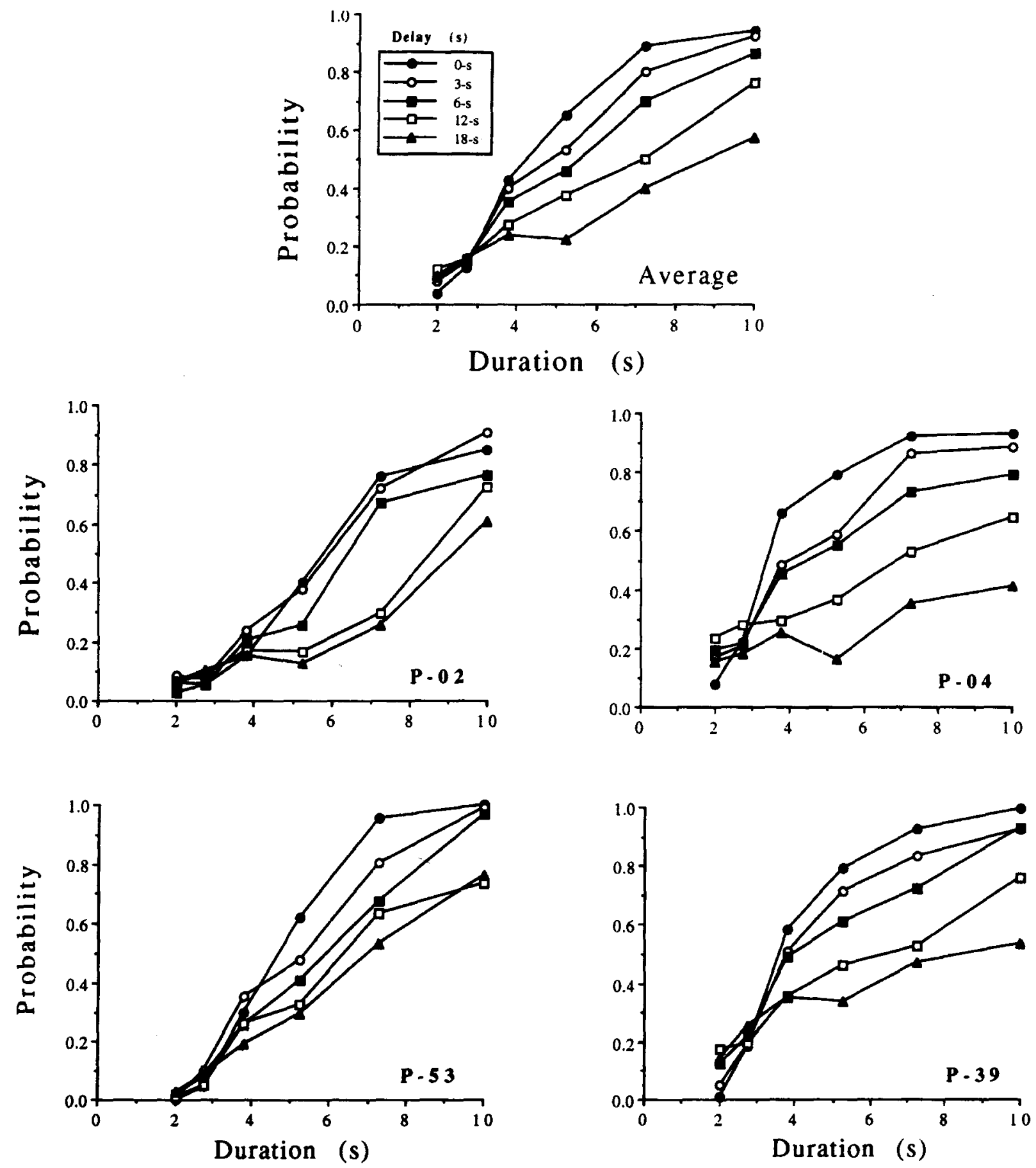

Figure 3. Psychometric functions relating the probability of responding to the long alternative to signal duration, with delay between signal ofiset and choice as the parameter. (The data were obtained from the second multiple-stimulus delay test.)

between durations and choices assessed the nature and extent of forgetting along the temporal dimension. Choice probabilities changed in an orderly way with changes in signal duration, and some control by signal duration, as assayed by slope measures, was evident at the longest retention intervals at a point when the functions clearly reflected a strong bias to "choose short." However, tem- poral control was not relinquished in an evenhanded way. The asymmetrical changes in the ogives, observed for all pigeons, are consistent with a model of delayed temporal control that holds that forgetting occurs along the temporal dimension (i.e., that subjects retain an analogical code for duration), that the remembered information changes systematically with time since the offset 

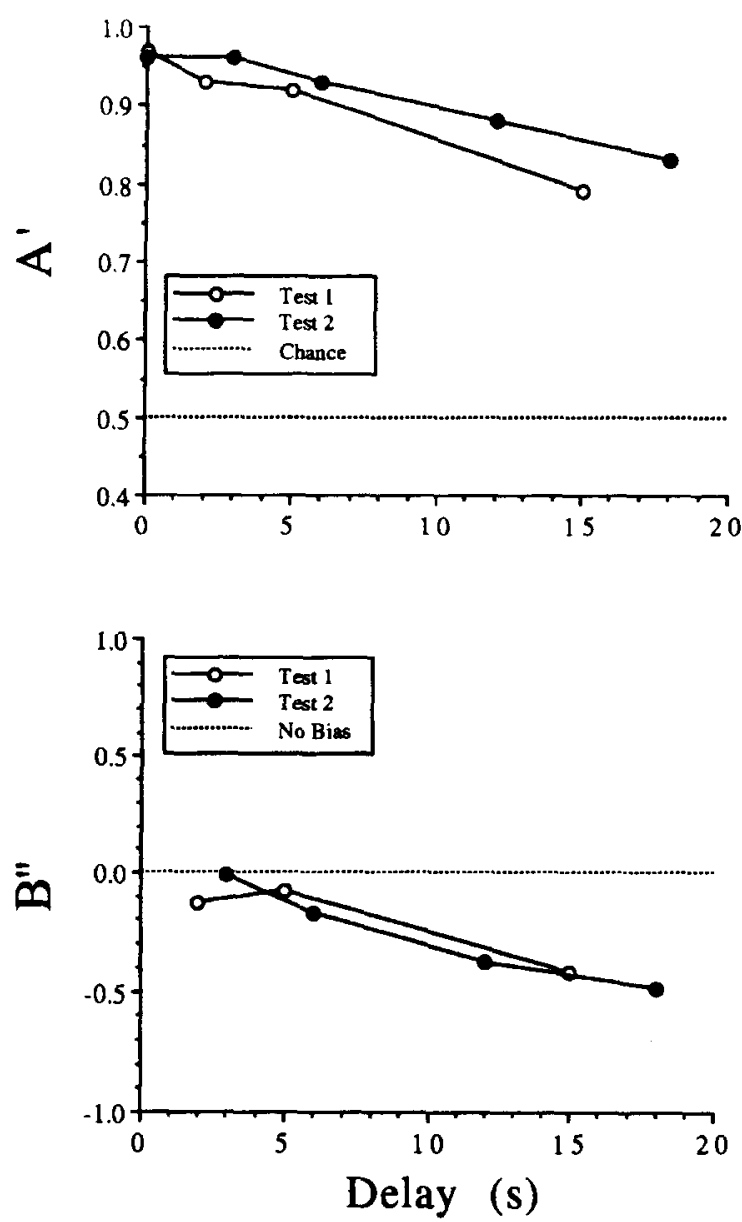

Figure 4. Measures of sensitivity $\left(A^{\prime}\right)$ and response bias $\left(B^{\prime \prime}\right)$ for the data shown in Figures 2 and 3. (See text for further details.)

of the carrier signal, and that subjects evaluate the duration of the signal retrospectively. This model, of course, is the subjective shortening hypothesis originally proposed by Spetch and Wilkie (1983).

\section{EXPERIMENT 2}

In Experiment 1, the probe (intermediate) stimuli were presented in extinction, and reinforcement was provided only when a pigeon chose correctly after the shortest or longest signal durations. This maintained generalization procedure provided a measure of dimensional control and afforded an estimate of the PSEs. In Experiment 2, the temporal bisection procedure was modified in a way first introduced by Stubbs (1968). Subjects were trained with a set of six signal durations; one choice was reinforced after the three shortest values, and a different choice was reinforced after the three longest values. Once the basic task was learned, delays between durations and choices were introduced.

This task differs in several ways from standard bisection procedures. First, the reinforcement contingencies should force subjects to set a choice criterion midway between the two durations on either side of the boundary between the short and long categories. This criterion is only loosely specified by the maintained generalization procedure. Second, the modified task included trials with different levels of discriminability. Extreme values (i.e., the shortest and longest durations) are more likely to be correctly identified than those adjacent to the category boundaries. The question was whether-and if so how-these differences would affect the psychophysics of remembered duration.

\section{Method}

Subjects and Apparatus. The subjects, deprivation regimen, and apparatus were the same as those described in Experiment 1.

Procedure. Experiment 2 was conducted immediately after the first multiple-stimulus delay test reported in Experiment 1, so no pretraining was required. The procedure was in most respects identical to the one described in Experiment 1. Trials began with the illumination of an amber light behind the center key; the light remained on for one of six durations-2.0,2.75,3.8, 5.25, 7.24, or $10.0 \mathrm{sec}$-and then the center keylight was turned off and the side keys were illuminated by red and green lights, with the position of each color varying randomly across trials. Trials were equally dis-
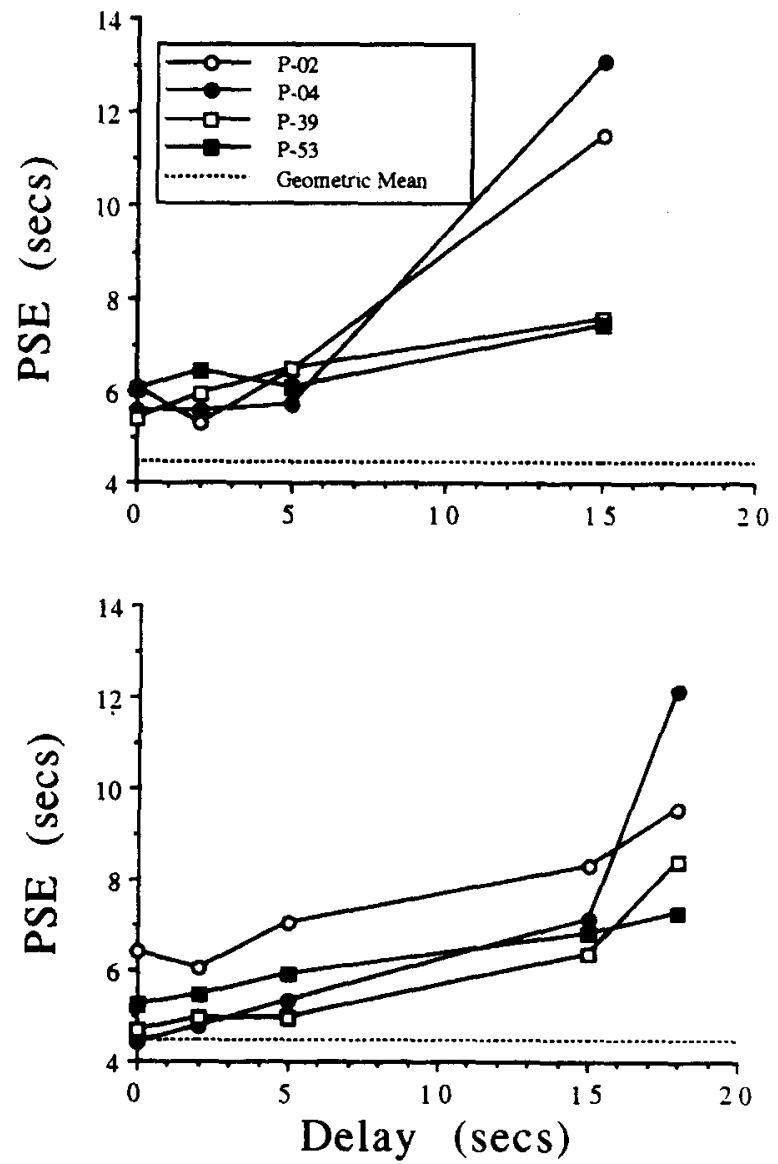

Figure 5. Points of subjective equality (PSEs) as a function of delay interval for each pigeon. PSEs were estimated from linear regressions fitted to the ogival data in Figures 2 and 3 . The data in the top panel are based on the ogival functions in Figure 2, and those in the bottom panel are from Figure 3. (See text for further details.) 
tributed among the six sample durations. A response to one key color was correct after the three shortest signal durations, and a response to the other key color was correct after the three longest durations. The sample duration to key-color mappings conformed to those used in Experiment 1; if a pigeon had been trained in Experiment 1 to peck red after the 2 -sec sample, it was required to peck red after the 2-, 2.75-, and 3.8-sec samples. Correct responses produced 3-sec access to mixed grain followed by a 30-sec ITI, during which all lights were off. Incorrect responses led directly to the ITI, and a noncorrection procedure was used. Sessions ended after 60 trials.

Ten sessions of training were given, with reinforcement after every correct choice. After this, the probability of reinforcement for correct responses was reduced to .50 and session length was increased to 108 trials. The birds received an additional 20 sessions of training with partial reinforcement of choices, and then delay testing was begun. During this phase, delays were sometimes interpolated between the offset of the center keylight and the onset of the side keylights on some trials; all the lights were off during delay intervals. One half of the trials were 0 -sec delay trials and the remaining trials were equally divided among delays of 2, 5, and $15 \mathrm{sec}$; sessions ended after 108 trials and delay testing lasted for 30 sessions.

\section{Results and Discussion}

Figure 6 shows psychophysical data for the last five sessions before delays were introduced. The format is comparable to Figures 2 and 3, showing the probability of responding to the long choice alternative as a function of signal duration. The curves are smooth and ogival, and the slopes appear steepest at the boundary between the short and long categories (between 3.8 and $5.25 \mathrm{sec}$ ), except for P-02.

Figure 7 presents the psychophysical functions from the delay test for each pigeon; the functions are based on the entire 30 sessions of delay testing. As in Experiment 1 , the slopes decreased with increasing delay between durations and choices. However, the asymmetries evident in Figures 2 and 3 are not so readily apparent, especially for Pigeons P-04 and P-39. In general, decreasing probabilities for longer durations were accompanied by increasing probabilities for shorter durations. As in Experiment 1, regressions were performed on $z$ score transforms of the independent and dependent measures. Slopes decreased significantly across delays $[F(3,9)=$ $12.11, p<.05]$. At the longest $(15 \mathrm{sec})$ delay, slopes were not significantly greater than zero for 2 of the birds (P-04 and P-39).

Although there appear to be qualitative differences in the psychophysics of temporal forgetting between Experiments 1 and 2, more precise measures of the changes are called for. Figure 8 presents measures of sensitivity $\left(A^{\prime}\right)$ and response bias $\left(B^{\prime \prime}\right)$ across delays for each pigeon. As in Figure 4, negative values of $B^{\prime \prime}$ indicate a bias to respond to the short alternative, and positive values indicate a bias favoring the long alternative.

The top panel shows the changes for $A^{\prime}$, indicating a decline in sensitivity to duration with increasing delay; the changes were significant $[F(3,9)=18.96, p<.05]$. The bottom panel shows the changes in $B^{\prime \prime}$. The tendency to choose the short alternative decreased with in-

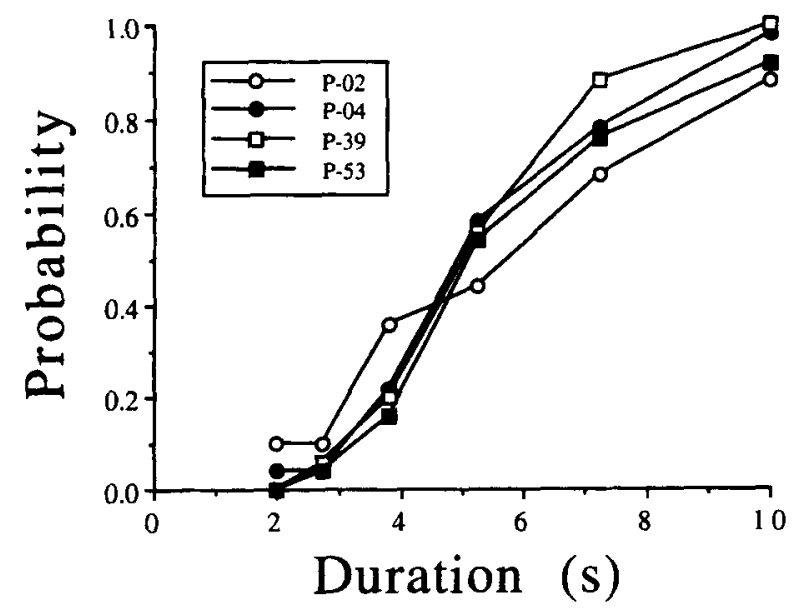

Figure 6. Psychometric functions relating the probability of responding to the long alternative to signal duration. Functions are shown for each pigeon after initial training with the modified procedure of Experiment 2.

creasing delay, contrary to the usual finding in experiments on delayed discrimination of duration; this change was statistically reliable $[F(3,9)=8.249, p<.05]$. Post hoc comparisons (Fisher $L S D$ ) indicated that values of $B^{\prime \prime}$ at the 0 -sec delay were significantly $(p<.05)$ less than those at all other delays; none of the remaining comparisons were significant.

Although the changes in bias shown in Figure 8 are the opposite of those typically observed and are different from those observed in Experiment 1 (Figure 4), the analysis was conducted at a fairly global level, which may mask differences at a more local level. The difficulty of the temporal discrimination varied from trial to trial due to the use of multiple values for the short and long categories. Extreme values ( 2 and $10 \mathrm{sec}$ ) of either category would likely be more discriminable than values adjacent to the category boundary $(3.8$ and $5.25 \mathrm{sec})$, or one step removed from the boundary $(2.75$ and $7.24 \mathrm{sec})$. Changes in bias in accord with subjective shortening of remembered duration may be related to the discriminability of the durations, and might be more evident at the category boundary than for stimuli far removed from the boundary. Accordingly, $A^{\prime}$ and $B^{\prime \prime}$ measures were calculated separately for easy ( 2 vs. $10 \mathrm{sec}$ ), medium ( $2.75 \mathrm{vs}$. $7.24 \mathrm{sec})$, and hard ( $3.8 \mathrm{vs} .5 .25 \mathrm{sec})$ discriminations.

Figure 9 shows the results of this analysis. The top panel shows $A^{\prime}$ across delays for the three levels of discrimination difficulty. The points are means of the 4 pigeons, and the bars represent standard errors of the means. The points are highest for the easy category, lowest for the hard category, and intermediate for the medium category. Values of $A^{\prime}$ for all categories decreased with increasing delay, and the functions appear very nearly parallel. A two-way analysis of variance (ANOVA) with delay and level of difficulty as the factors showed that the main effects of delay $[F(3,9)=$ 

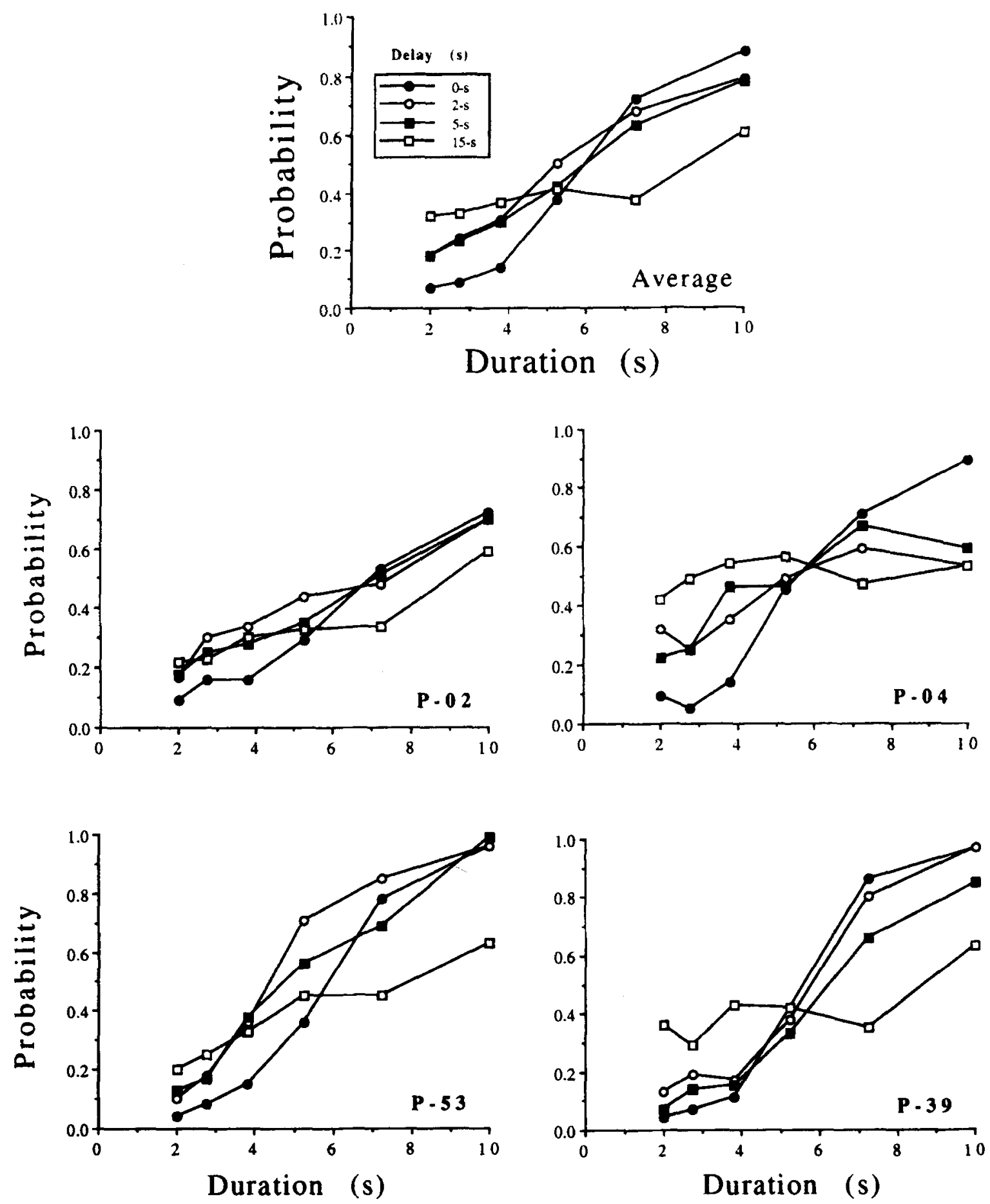

Figure 7. Psychometric functions relating the probability of responding to the long alternative to signal duration, with delay between signal offset and choice as the parameter.

$16.17, p<.05]$ and level of difficulty $[F(2,6)=42.87$, $p<.05]$ were significant; the interaction of these two factors was not significant $[F(6,18)=2.06, p>.05]$.

The bottom panel of Figure 8 shows the changes for the bias measure, $B^{\prime \prime}$, for each level of difficulty and delay. There is very little change in bias at any level of difficulty, except between the $0-\mathrm{sec}$ and 2 -sec delays.
A two-way ANOVA was carried out on the $B^{\prime \prime}$ data, and neither the main effects nor the interaction was significant.

Although statistical analysis indicates that the changes in bias across delays are nonsignificant, one feature of the patterns merits further examination. Figure 9 shows that all the pigeons displayed a strong bias to respond 

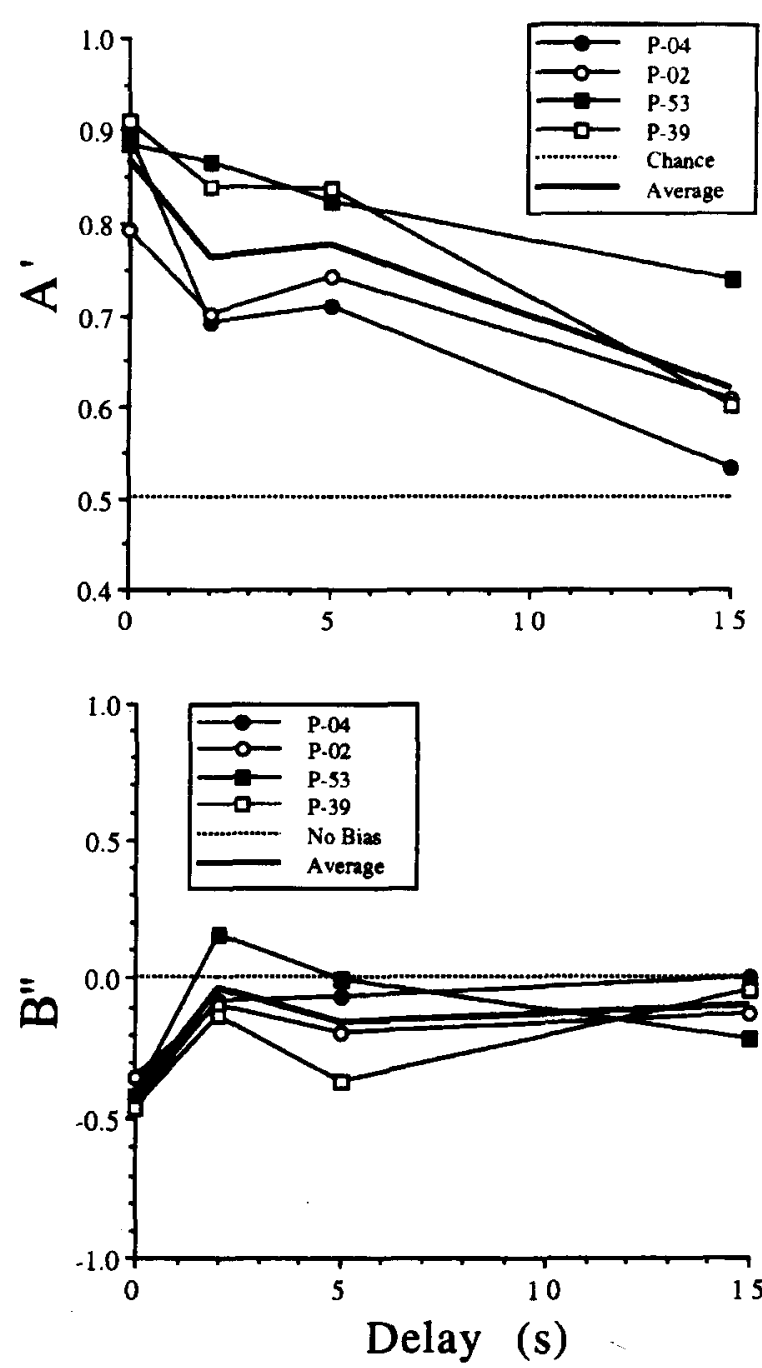

Figure 8. Measures of sensitivity $\left(A^{\prime}\right)$ and response bias $\left(B^{\prime \prime}\right)$ across delays for the data shown in Figure 6. (See text for further details.)

"short" for the medium and hard categories at the 0 -sec delay. This result is surprising because choices were differentially reinforced for all durations, and an "ideal" observer should establish a criterion for responding midway between the values adjacent to the category boundary.

Figure 10 provides further information about this asymmetry. The figure shows mean probability of correct scores for short and long durations for each level of difficulty; the bars are standard errors of the means. The differences in accuracy increased with decreasing difference between the short and long durations of the pairs. When the discrimination involved durations at the category boundary $(3.8$ and $5.25 \mathrm{sec}$ ), the differences in accuracy were strikingly large and like those observed when choices are delayed from the presentation of the durations (i.e., the choose-short effect). Each pair of scores was transformed to a single difference score measure reflecting accuracy on short trials minus accuracy on long trials. A one-way ANOVA with problem diffi- culty as the factor was computed on these measures. The $F$ ratio was significant $[F(2,6)=62.07, p<.05]$. Post hoc comparisons revealed that all the scores were significantly different from each other $(p<.05)$.

The modified procedure of Experiment 2 appeared to alter the psychophysics of remembered duration. By comparison with Experiment 1, the decreases in temporal control across delays were fairly unbiased; that is, there was little evidence for a subjective shortening process. It should be noted that the manipulations reported as Experiment 2 were conducted between the first and second exposure to the maintained generalization procedure reported as Experiment 1, constituting an ABA design. Both determinations of the maintained generalization task produced changes in the psychophysical functions that appear qualitatively different from those obtained in Experiment 2.

One plausible cause of the difference is a change in subjects' choice criteria. As suggested above, perhaps the reinforcement contingencies incorporated in the modified method of constant stimuli used in Experi-
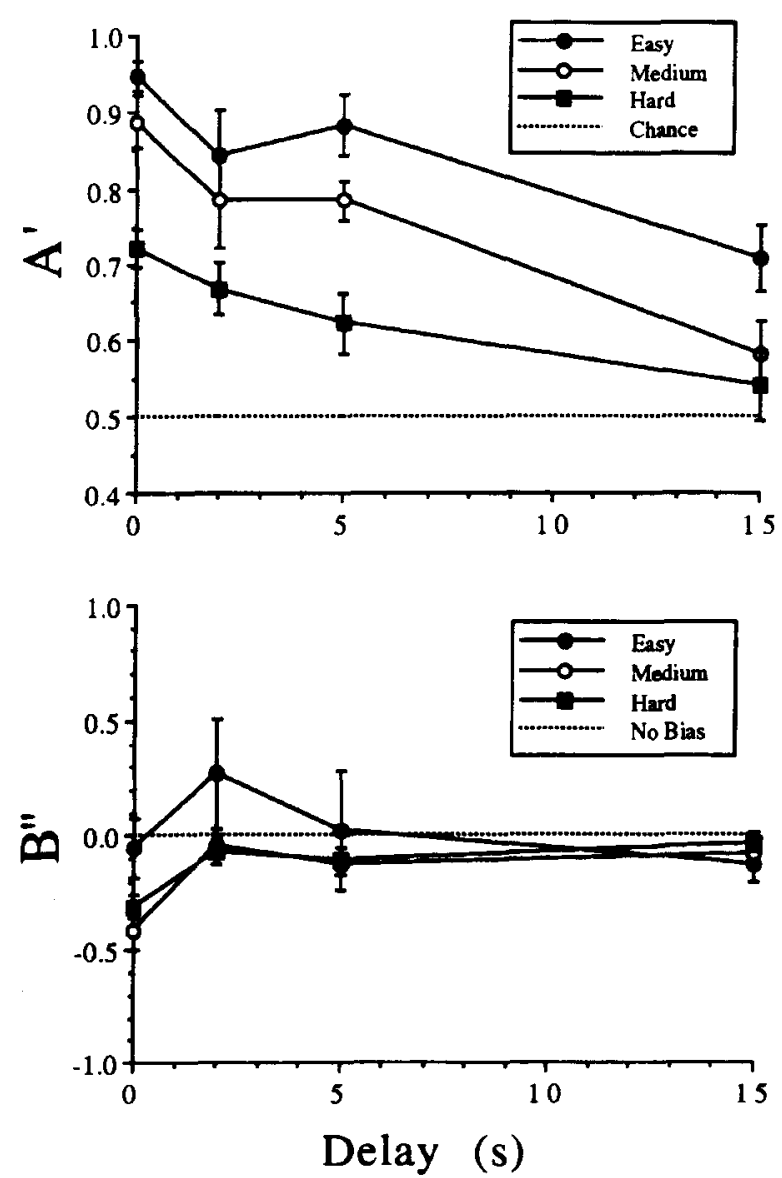

Figure 9. Measures of sensitivity $\left(A^{\prime}\right)$ and response bias $\left(B^{\prime \prime}\right)$ across delays. Measures were calculated separately for easy ( 2 vs. 10 sec), medium ( $2.75 \mathrm{vs} .7 .24 \mathrm{sec})$, and hard ( $3.8 \mathrm{vs} .5 .25 \mathrm{sec})$ discriminations. The data are averaged over 4 pigeons; the bars represent plus and minus one standard error of the means. 


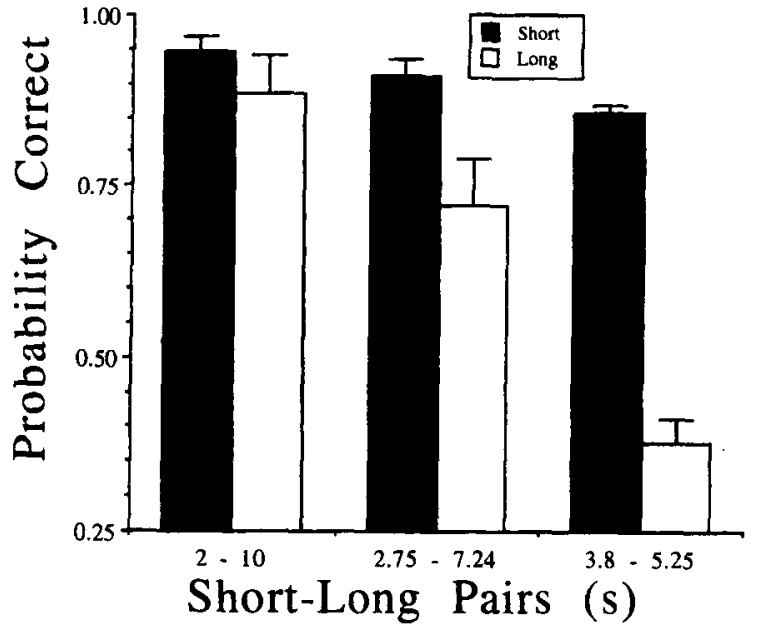

Figure 10. Accuracy (probability correct) at zero delay after short and long signals involving easy, medium, and hard discriminations, as defined in Figure 9. The data are averaged over 4 pigeons; the bars represent one standard error of the means.

ment 2 shifted the criterion in a direction that produced a more evenhanded evaluation of remembered duration. Alternatively, the procedure of Experiment 2 could be viewed as an MTO mapping arrangement under which three sample durations were mapped onto each choice response. This arrangement typically encourages a "common coding" strategy (e.g., Urcuioli, Zentall, JacksonSmith, \& Steirn, 1989) whereby the different samples are represented by a single memory code; such strategies are held to be incompatible with analogical coding of duration (e.g., Grant \& Spetch, 1993), as required by the subjective shortening model. The critical test of this notion would involve a comparison of retention functions for the last few sessions of the procedure reported in Experiment 2 and the first few sessions of testing after the birds were returned to the maintained generalization procedure (second test series of Experiment 1), where the birds evidently reverted to the analogical coding strategy. However, the subjects were given approximately 30 sessions of retraining on the maintained generalization test before retention tests were conducted, so meaningful comparisons are not possible.

The disparity in accuracy on short- and long-signal trials at the 0 -sec delay (Figure 10 ) is surprising. It could be argued that the disparity was due to a lack of correspondence between physical and psychological scales. However, sample durations were spaced equal log steps apart, and, on the basis of fairly extensive knowledge of temporal psychophysics (e.g., Gibbon, 1986), this spacing should have produced equally discriminable changes along the temporal continuum. A second possibility is that the bias was caused by the introduction of delays between durations and choice whereby the intermingling of trials with and without delays produced a criterion shift toward longer durations. However, examination of the data from the last five sessions before delay testing
(Figure 6) reveals the identical pattern of accuracy differences.

Inspection of the bisection data of Figure 5 suggests a final possibility. The top panel of Figure 5 shows that bisection points at the 0 -sec delay were between 5.5 and $6 \mathrm{sec}$ for all the birds. Thus, the most difficult long duration of Experiment $2(5.25 \mathrm{sec})$ was below the bisection point of the previous condition; carryover from the prior condition thus could have contributed to the birds' biased classification of signals near the category boundary. Once again, however, the birds received extensive training under the task reported in Experiment 2, which should have minimized or eliminated carryover effects.

\section{GENERAL DISCUSSION}

These experiments demonstrate that pigeons' choices are sensitive to variations in signal duration when choice opportunities are delayed from the signal. This finding is consistent with models of temporal memory that hold that subjects retain information that bears an analogical correspondence to signal duration (Spetch \& Rusak, 1992; Spetch \& Wilkie, 1983; Wilkie \& Willson, 1990). When test stimuli intermediate to the shortest and longest signals were presented in extinction (Experiment 1), the changes in the psychometric functions and derived measures (PSEs) were consistent with a subjective shortening process; probabilities of choosing "long" decreased for longer signal durations, but did not increase for shorter signal durations. A modified version of the method of constant stimuli (Experiment 2) produced comparable decreases in sensitivity, but the changes in the ogives were qualitatively different from those observed in the first experiment; stimulus control decreased equally after shorter and longer durations.

We first consider these findings in the context of quantitative accounts of timing. The analogical coding position is consistent with a leading theory of timing in nonhuman animals, scalar expectancy theory (SET; e.g., Gibbon \& Church, 1984). The theory holds that temporal control is mediated by a dedicated informationprocessing apparatus consisting of several functional components, including a pacemaker, switch, accumulator, and comparator, that interact with working and reference memory (see Church, 1984, for a detailed description). Time is represented in this system by the number of pulses received by the accumulator. Pulse number values are compared with remembered values stored in reference memory, and the animal's observed behavior (e.g., pecking the red or green comparison stimulus) is based on the outcome of this comparison. Numerous experiments employing many different timing tasks have produced results consistent with this construction of the timing process (e.g., see a review by Church, 1989). Although analogical encoding of duration is seemingly required by SET, the theory has not been elaborated to account for the way in which the encoded information changes over time, when it must be 
retained in working memory and used to mediate responses in delayed discrimination experiments of the kind described in this article and elsewhere.

Killeen and Fetterman (1988) explained the chooseshort effect in terms of their behavioral theory of timing $(\mathrm{BeT})$. They suggested that subjects normally base choices ("short" vs. "long") on behavioral states conditioned to the different response alternatives. When delays are placed between durations and choices, subjects exit these conditioned states as an exponentially decreasing function of the delay interval. When the choices are presented at the end of the delay and the subject is not in one of the conditioned states, it chooses with a biased probability, with the bias favoring the short response alternative. This model provided a very good quantitative account of data published by Spetch and Wilkie (1982) and by Kraemer et al. (1985). However, the model does not specify the source of the response bias correlated with unconditioned response states, and this bias is the crux of the choose-short effect.

Quantitative models of timing do not provide a completely satisfactory account of the observed asymmetries of delayed temporal control, but perhaps such an account should not be demanded of theories developed to explain contemporaneous timing effects. Thus, we turn to quantitative models of animal working memory. Although research and theory on temporal and nontemporal animal working memory historically have emphasized qualitative differences, several quantitative accounts have recently been proposed (e.g., Harnett, McCarthy, \& Davison, 1984; McCarthy \& White, 1987; Wixted, 1989; Wixted \& Ebbesen, 1991). Much of this research has concerned the form of the forgetting function (e.g., exponential vs. hyperbolic) and variables that systematically affect the parameters of the forgetting functions. This work has organized a large body of literature on animal working memory (Wixted, 1989), but it has been applied only to experiments with nontemporal stimuli (e.g., hues, forms), in which stimulus control dissipates equally after each sample type. One of these functions could easily be fit to overall accuracy data from the present experiment, but such an account would obscure the differential changes for different sample durations.

In contrast, Staddon (1984) has proposed a quantitative description of timing and remembering that places time discrimination and memory in a common framework. Staddon argued that both timing and remembering could be construed as involving a discrimination of event saliencies, where the salience of an event is directly related to its duration (longer events are more salient than shorter events) and inversely related to its recency (recent events are more salient than events in the more distant past). Duration discrimination thus becomes a discrimination of event saliencies (see Wilkie, 1987, for supporting data). This model affords a natural link between treatments of delayed matching-to-sample that picture the situation as involving a discrimination of relative recencies (e.g., D'Amato, 1973) and temporal discrimination tasks.
Equation 1 quantifies these principles, giving the salience $(S)$ of an event as a function of the difference between time since the event ended and the time since it began,

$$
S=A\left(t_{1}^{m}-t_{2}^{m}\right), m<0,
$$

where $t_{1}$ is how long ago the event ended, $t_{2}$ is how long ago the event began, $A$ is a constant of proportionality, and the exponent, $m$, determines how rapidly salience declines as a function of event age. Equation 1 specifies that the initial changes in the salience of a delayed event are rapid, but occur more slowly with further increases in the delay (increases in $t_{1}$ ).

Table 1 shows the outcome of computations based on this model applied to a set of sample durations and delays used in the present experiments. Because there is some latency in responding to the choices after the offset of the sample stimulus, we assume that $t_{1}$ is always greater than zero, even on zero-delay trials. The calculations assume a minimal value of $0.5 \mathrm{sec}$ for $t_{1}$, and a value of -0.10 for $m$. The constant of proportionality, $A$, was set to one because it does not bear on understanding the predictions of the model.

The transformation of nominal durations in Table 1 has several features that accord with data on temporal memory. First, the differences between transformed sample durations decrease with increases in delay, which should result in lower overall discriminability (a decrease in the slopes of the psychometric function). Second, salience decreases with increases in delays; if delayed temporal discriminations are based on event saliencies, then delayed sample durations will appear less salient (shorter) than contemporaneous sample durations, producing a tendency to select the alternative associated with the less salient sample. These changes are analogous to a subjective shortening process. Finally, although the differences are small, comparisons of adjacent pairs of transformed values at the 0 -sec delay reveal that the subjective difference is larger for shorter than for longer durations; at nonzero delays the opposite pattern is found-that is, subjective differences are larger for longer than for shorter durations. The former result is consistent with the asymmetries in accuracy for short and long signal pairs shown in Figure 10. Staddon's model provides a compromise between analogical and categorical accounts. His model is compatible with "trace-

Table 1

Nominal and Transformed Durations (in Seconds) as a Function of Delay (in Seconds) Between the Duration and Choice

\begin{tabular}{ccccccc}
\hline & \multicolumn{7}{c}{ Nominal Duration } \\
\cline { 2 - 7 } Delay & 2 & 2.75 & 3.8 & 5.25 & 7.24 & 10 \\
\hline 0 & .139 & .168 & .197 & .225 & .251 & .277 \\
2 & .042 & .057 & .074 & .092 & .119 & .133 \\
5 & .020 & .028 & .039 & .051 & .065 & .081 \\
15 & .007 & .010 & .015 & .020 & .027 & .036
\end{tabular}

Note-The transformation is based on Staddon's (1984) model of timing and remembering. See text for details. 
strength" (Roberts \& Grant, 1976) views of working memory for metathetic stimuli (e.g., hues) and affords an explanation of delayed discriminations of the prothetic continuum of duration.

A shortcoming of Staddon's approach is the lack of specificity about the role of nontemporal factors. For instance, the model does not address the issue of qualitative differences in memories (i.e., what is remembered), focusing only on the efficacy of control by events in the past. Work on temporal memory, for example, suggests that procedural changes can alter the nature of the inferred memory code (Grant \& Spetch, 1991). The model also does not include an explicit decision process to guide subjects' choices based on event saliencies; such a process is implicit in this approach, but would need to be made explicit to provide a realistic test of the model and to account for the differences in results between Experiments 1 and 2 .

Recent work on temporal memory (e.g., Grant \& Spetch, 1993, 1994; Santi et al., 1993) suggests that pigeons may be induced to adopt nontemporal coding strategies. The methods for inducing such strategic changes involve substantial modifications of the temporal DSMTS originally studied by Spetch and Wilkie $(1982,1983)$. Although these newer findings are important and provide additional evidence for the flexibility of memory coding, the original biased forgetting result remains an extremely robust working-memory phenomenon, deserving of continued attention.

\section{REFERENCES}

Allan, L. G., \& Gibbon, J. (1991). Human bisection at the geometric mean. Learning \& Motivation, 22, 39-58.

Brown, P. L., \& Jenkins, H. M. (1968). Autoshaping of the pigeon's keypeck. Journal of the Experimental Analysis of Behavior, 11, 1-8.

$\mathrm{C}_{\mathrm{HURCH}}$, R. M. (1980). Short -term memory for time intervals. Learning \& Motivation, 11, 208-219.

Church, R. M. (1984). The internal clock. In J. Gibbon \& L. Allan (Eds.), Timing and time perception (Annals of the New York Academy of Sciences, Vol. 423, pp. 566-582). New York: New York Academy of Sciences.

Church, R. M. (1989). Theories of timing behavior. In S. Klein \& R. R. Mowrer (Eds.), Contemporary learning theories: Instrumental conditioning theory and the impact of biological constraints on learning (pp. 41-71). Hillsdale, NJ: Erlbaum.

Church, R. M., \& DeluTy, M. Z. (1977). Bisection of temporal intervals. Journal of Experimental Psychology: Animal Behavior Processes, 3, 216-228.

D'Amato, M. (1973). Delayed matching and short-term memory in monkeys. In G. H. Bower (Ed.), The psychology of learning and motivation: Advances in research and theory (Vol. 7, pp. 227-269). New York: Academic Press.

Fetterman, J. G., Dreyfus, L. R., \& Stubbs, D. A. (1993). Discrimination of duration ratios by pigeons (Columba livia) and humans (Homo sapiens). Journal of Comparative Psychology, 107, 3-11.

Fetterman, J. G., \& Killeen, P. R. (1992). Time discrimination in Columba livia and Homo sapiens. Journal of Experimental Psychology: Animal Behavior Processes, 18, 80-94.

Fetterman, J. G., \& MACEWEN, D. (1989). Short-term memory for responses: The "choose small" effect. Journal of the Experimental Analysis of Behavior, 52, 311-324.

GibBon, J. (1986). The structure of subjective time: How time flies. In G. H. Bower (Ed.), The psychology of learning and motivation: $A d$ - vances in research and theory (Vol. 20, pp. 105-135). San Diego, CA: Academic Press.

GibBon, J. (1991). Origins of scalar timing. Learning \& Motivation, 22, 3-38.

GibBon, J., \& ChURCH, R. (1984). Sources of variability in an information processing theory of timing. In H. L. Roitblat, T. G. Bever, \& H. S. Terrace (Eds.), Animal cognition (pp. 465-488). Hillsdale, NJ: Erlbaum.

Grant, D. S., \& Spetch, M. L. (1991). Pigeons' memory for event duration: Differences between choice and successive matching tasks. Learning \& Motivation, 22, 180-199.

Grant, D. S., \& SPETCH, M. L. (1993). Analogical and nonanalogical coding of samples differing in duration in a choice-matching task in pigeons. Journal of Experimental Psychology: Animal Behavior Processes, 19, 15-25.

Grant, D. S., \& SpeTCH, M. L. (1994). Mediated transfer testing provides evidence for common coding of duration and line samples in many-to-one matching in pigeons. Animal Learning \& Behavior, 22, 84-89.

GrIER, J. G. (1971). Nonparametric indexes for sensitivity and bias: Computing formulas. Psychological Bulletin, 75, 424-429.

Harnett, P., McCarthy, D., \& Davison, M. (1984). Delayed signal detection, differential reinforcement, and short-term memory in the pigeon. Journal of the Experimental Analysis of Behavior, 42, 87111.

Honig, W. K., \& SPETCH, M. (1988). Short-term memory for rate of alternation in the pigeon. Bulletin of the Psychonomic Society, 26, 152-154.

Honig, W. K., \& Thompson, R. K. R. (1982). Retrospective and prospective processing in animal working memory. In G. H. Bower (Ed.), The psychology of learning and motivation: Advances in research and theory (Vol. 16, pp. 239-283). Orlando, FL: Academic Press.

Killeen, P. R., \& Fetterman, J. G. (1988). A behavioral theory of timing. Psychological Review, 95, 274-295.

KraEmer, P. J. (1991). Absence of immediate transfer of training of duration symbolic-matching-to-sample in pigeons. Animal Learning \& Behavior, 19, 276-282.

Kraemer, P. J., Mazmanian, D. S., \& Roberts, W. A. (1985). The choose-short effect in pigeon memory for stimulus duration: Subjective shortening versus coding models. Animal Learning \& Behavior, 13, 349-354.

MCCARTHY, D., \& WHITE, K. G. (1987). Behavioral models of delayed detection and their application to the study of memory. In M. L. Commons, J. E. Mazur, J. A. Nevin, \& H. Rachlin (Eds.), Quantitative analyses of behavior: The effect of delay and of intervening events on reinforcement value (pp. 29-54). Hillsdale, NJ: Erlbaum.

RoBerTs, W. A., \& GRANT, D. S. (1976). Studies of working memory in the pigeon using the delayed-matching-to-sample procedure. In D. L. Medin, W. A. Roberts, \& R. T. Davis (Eds.), Processes of animal memory (pp. 79-112). Hillsdale, NJ: Erlbaum.

Santi, A., Bridson, S., \& Ducharme, M. J. (1993). Memory codes for temporal and nontemporal samples in many-to-one matching by pigeons. Animal Learning \& Behavior, 21, 120-130.

Spetch, M. L., \& Rusak, B. (1992). Time present and time past. In W. K. Honig \& J. G. Fetterman (Eds.), Cognitive aspects of stimulus control (pp. 47-67). Hillsdale, NJ: Erlbaum.

SPETCH, M. L., \& Sinha, S. S. (1989). Proactive effects in pigeons' memory for event duration: Evidence for analogical retention. Journal of Experimental Psychology: Animal Behavior Processes, 15, 347-357.

SPETCH, M. L., \& Wilkie, D. M. (1982). A systematic bias in pigeons' memory for food and light durations. Behaviour Analysis Letters, 2 , 267-274.

SPETCH, M. L., \& Wilkie, D. M. (1983). Subjective shortening: A model of pigeons' memory for event duration. Journal of Experimental Psychology: Animal Behavior Processes, 9, 14-30.

STADDON, J. E. R. (1984). Time and memory. In J. Gibbon \& L. Allan (Eds.), Timing and time perception (Annals of the New York Academy of Sciences, Vol. 423, pp. 322-334). New York: New York Academy of Sciences. 
StubBs, A. (1968). The discrimination of stimulus duration by pigeons Journal of the Experimental Analysis of Behavior, 11, 223-238.

StUBBS, D. A. (1976), Response bias and the discrimination of stimulus duration. Journal of the Experimental Analysis of Behavior, 25, 243-250.

Urcuioli, P. J., Zentall, T. R., Jackson-Smith, P., \& Steirn, J. (1989). Evidence for common coding in many-to-one matching: Retention, intertrial interference, and transfer. Journal of Experimental Psychology: Animal Behavior Processes, 15, 264-273.

WILKIE, D. M. (1987). Stimulus intensity affects pigeons' timing behavior: Implications for an internal clock model. Animal Learning \& Behavior, 15, 35-39.
Wilkie, D. M., \& WiLlson, R. J. (1990). Discriminal distance analysis supports the hypothesis that pigeons retrospectively encode event duration. Animal Learning \& Behavior, 18, 124-132.

WIXTED, J. T. (1989). Nonhuman short-term memory: A quantitative reanalysis of selected findings. Journal of the Experimental Analysis of Behavior, 52, 409-426.

Wixted, J. T., \& EbBesen, E. B. (1991). On the form of forgetting. Psychological Science, 2, 409-415.

(Manuscript received March 4, 1994;

revision accepted for publication April 20, 1994.) 